\title{
Impact of Metabolic Syndrome on Response to Medical Treatment of Benign Prostatic Hyperplasia
}

\author{
Ali Cyrus, Ali Kabir ${ }^{1}$, Davood Goodarzi, Afsaneh Talaei ${ }^{2}$, Ali Moradi $^{3}$, Mohammad Rafiee ${ }^{4}$, \\ Mehrdad Moghimi $^{5}$, Elham Shahbazi ${ }^{6}$, Elaheh Farmani ${ }^{6}$
}

Department of Urology, Arak University of Medical Sciences, Arak, ${ }^{1}$ Minimally Invasive Surgery Research Center, Iran University of Medical Sciences, and Department of Epidemiology, Faculty of Public Health, Shahid Beheshti University of Medical Sciences, Tehran, ${ }^{2}$ Department of Endocrinology, Arak University of Medical Sciences, Arak, ${ }^{3}$ Asadabad Health and Treatment Network, Hamedan University of Medical Sciences, Hamadan, and Department of Epidemiology, Faculty of Public Health, Shahid Beheshti University of Medical Sciences, Tehran, ${ }^{4}$ Department of Statistics, Arak University of Medical Sciences, Arak, ${ }^{5}$ Department of Surgery, Shahid Beheshti University of Medical Sciences, Tehran, ${ }^{6}$ Arak University of Medical Sciences, Arak, Iran

Purpose: To investigate the effect of metabolic syndrome (MetS) on the response to medical therapy of benign prostatic hyperplasia (BPH) after a 3-month period of treatment. Materials and Methods: This was a cohort study of 100 patients, 47 with MetS and 53 without MetS, referred to either the primary care unit or referral hospital with BPH who had moderate lower urinary tract symptoms of prostate involvement and were candidates for medical treatment. Our main outcome was response to medical treatment with prazosin $1 \mathrm{mg}$ twice a day and finasteride $5 \mathrm{mg}$ daily in patients with BPH on the basis of International Prostate Symptom Score (IPSS). Multivariate analysis of covariance was used to compare $\mathrm{BPH}$ treatment response in patients with and without MetS before and after receiving treatment.

Results: The mean volume of the prostate was significantly higher in MetS patients than in patients without MetS $(57 \pm 32.65 \mathrm{~mL}$ compared with $46.00 \pm 20.19 \mathrm{~mL}, \mathrm{p}=0.036)$. The control group demonstrated an 11-unit reduction in IPSS, whereas those with MetS showed a reduction in the symptom score of only 6 units $(p<0.001)$. Regarding the components of MetS separately, triglyceride $(p<0.001)$, fasting blood sugar $(p=0.001)$, and waist circumference $(\mathrm{p}=0.028)$ significantly affected the clinical progression of $\mathrm{BPH}$. The observational nature of this study may be a limitation in comparison with an interventional study.

Conclusions: The results of the present study showed that MetS can negatively affect the response to medical treatment of $\mathrm{BPH}$. Therefore, it is necessary to consider MetS in selecting patients with $\mathrm{BPH}$ for drug therapy.

Keywords: Finasteride; Lower urinary tract symptoms; Metabolic syndrome X; Prazosin; Prostatic hyperplasia

This is an Open Access article distributed under the terms of the Creative Commons Attribution Non-Commercial License (http://creativecommons.org/licenses/by-nc/3.0) which permits unrestricted non-commercial use, distribution, and reproduction in any medium, provided the original work is properly cited.

\section{Article History:}

received 23 July, 2014

accepted 28 October, 2014
Corresponding Author:

Ali Kabir

Nikan Health Researchers

Institute, Unit 9, No. 1, 3rd floor, 3rd Bahar Alley, Ashrafi Isfahani

Highway, Poonak Square,

Tehran, Iran

TEL: +98-21-44468645

FAX: +98-21-44476796

E-mail: aikabir@yahoo.com

\section{INTRODUCTION}

Benign prostatic hyperplasia (BPH), which develops in the transitional zone of the prostate, is the most common benign tumor in men [1]. Although the etiology of $\mathrm{BPH}$ is still unclear, research has shown that BPH is a multifactorial endocrine-related disease. Dihydrotestosterone and old age are the most important risk factors for $\mathrm{BPH}$, because the prostate is much more sensitive to androgens in older men [2].

According to the most trustworthy epidemiologic study regarding $\mathrm{BPH}$ prevalence in recent years, in the United States in 2000, BPH was the main reason for 4.4 million references to clinics, 117,000 visits to the Emergency 
Department, and 105,000 admissions in hospitals [3]. Pathologic study of autopsy samples has revealed histological signs of BPH in $42 \%$ of men within the age range of 51 to 60 years, which increases to $85 \%$ of men older than 80 years [4]. In studies investigating the association of $\mathrm{BPH}$ and waist circumference, it has been observed that obesity may cause BPH owing to endocrine alterations. Obesity can elevate estrogen and lower testosterone levels, which can increase the risk of BPH in obese people [5]. The following disorders have been proposed as $\mathrm{BPH}$ risk factors: family history, ethnicity, smoking, diabetes mellitus (DM) type II, hypertension, obesity, high-density lipoprotein cholesterol (HDL), and high insulin levels [6].

Lower urinary tract symptoms, which may be related to prostatic diseases such as BPH, are common in men of different ethnicities and demographic characteristics [7]. Epidemiologic, histopathological, molecular pathological, and clinical trials have recently showed that metabolic syndrome (MetS) and its components may play roles in the pathogenesis of $\mathrm{BPH}$ and prostate cancer [8].

MetS is a multifactorial disease that has been known by various names such as insulin-resistance syndrome, plurimetabolic syndrome, and deadly quartet and that has a high prevalence worldwide [9]. The clinical manifestations of MetS include obesity, low HDL, high blood pressure, high fasting blood sugar (FBS), high triglyceride (TG), and insulin resistance, which may further cause more severe disorders including hyperinsulinemia and glucose metabolism disorders.

Three studies in India have shown that the prevalence of MetS was $13 \%$ in Jaipur and $11.2 \%$ to $41 \%$ in Chennai [10]. It has been reported that age had a significant correlation with MetS prevalence and that $43.5 \%$ of people between the ages of 60 and 69 years are afflicted with it [11]. This disorder is more prevalent in adults and $25 \%$ of the United States adult population has MetS [12]. This number is lower in South Korea, where $14.2 \%$ of men and $17.7 \%$ of women are afflicted [13]. In recent years, some have investigated the associations between MetS components, anthropometric measurements, volume of the prostate, lower urinary tract symptoms, and BPH [14-16].

The Tehran Lipid and Glucose Study showed that MetS prevalence in 3- to 9-year-old Iranian children was $0.9 \%$. The prevalence of MetS in overweight, moderately overweight, and normal-weight children was $8.9 \%, 0.8 \%$, and $0.1 \%$, respectively [17]. Another Iranian study showed that MetS prevalence was $32.1 \%$, and that its prevalence among women was significantly higher than among men. That study also showed that MetS prevalence had an ascending pattern with increasing age and body mass index (BMI) [18].

On the basis of these previous studies demonstrating the association between MetS and BPH and the importance of MetS on BPH prognosis and cure rate, we decided to compare $\mathrm{BPH}$ clinical progression in patients with $\mathrm{BPH}$ and MetS who were candidates for medical treatment and in those without MetS. Differences observed between the clinical response and progression of these two groups would help to use MetS or its components as prognostic factors in predicting the outcome of $\mathrm{BPH}$ medical treatment.

\section{MATERIALS AND METHODS}

\section{Target population}

In this cohort study, patients with moderate symptoms of $\mathrm{BPH}$ who were chosen for medical treatment in Vali Asr Hospital and Urologic Clinics in Arak (located in the central part of Iran) were enrolled. On the basis of their medical history, physical examination, anthropometric measurements, and laboratory data, the patients were divided into two groups: patients with and those without MetS. The estimated sample size was 99 based on $\alpha=0.05$, power $=0.80$, standard deviation (SD) $=5$, and 2.85 units of difference in International Prostate Symptom Score (IPSS) as clinically significant by comparing two means formula. On the basis of previous estimations, we expected to see almost one-half of the population with MetS; hence, the samples were selected by the convenience method.

\section{Data collection}

All details and the purpose of the project were completely explained to all patients and signed informed consent was obtained. A comprehensive medical history including general health status, past history, drug history, signs and symptoms, and underlying diseases was taken.

IPSS, waist circumference, and blood pressure were documented for all patients. Laboratory assessments included complete blood count, blood urea nitrogen, creatinine, FBS, TG, cholesterol, HDL, low-density lipoprotein cholesterol (LDL), urine analysis, urine culture, and prostate-specific antigen (PSA). Sonograms of the urinary system and prostate and digital rectal examinations were also performed. On the basis of these findings, patients with an IPSS of 8 to 19 who did not have secondary complications of $\mathrm{BPH}$ became candidates for medical treatment and were treated under observation of urologists in a 3-month period with an alpha-blocker (prazosin $1 \mathrm{mg}$ twice a day) and finasteride $5 \mathrm{mg}$ (daily), which is routine treatment for $\mathrm{BPH}$ patients. All cases were visited by urologists monthly and were evaluated again for the response to treatment and IPSS after 3 months. Missed or excluded patients were replaced with similar ones.

\section{Measurements}

Because the World Health Organization (WHO) criteria for the diagnosis of MetS include urinary microalbumin in addition to the International Diabetes Federation and Adult Treatment Panel III criteria, we decided to use these more comprehensive criteria for MetS. According to the WHO, patients with 3 of the following findings have MetS: blood pressure $\geq 140 / 90 \mathrm{mmHg}, \mathrm{TG} \geq 150 \mathrm{mg} / \mathrm{dL}, \mathrm{HDL}<35 \mathrm{mg} / \mathrm{dL}$, waist circumference $>90 \mathrm{~cm}, \mathrm{FBS} \geq 100 \mathrm{mg} / \mathrm{dL}$, and microalbumin $\geq 20 \mu \mathrm{g} / \mathrm{min}$.

Regarding clinical progression of BPH before and after 
treatment, IPSS and lower urinary tract symptoms were used as follows: incomplete emptying or postvoiding residue (PVR), frequency, intermittency, urgency, weak stream, straining, nocturia, and hesitancy. We subdivided the IPSS into the obstructive score (sum of PVR, intermittency, weak stream, and straining $=0-20$ ) and the irritative score (sum of frequency, urgency, and nocturia $=0-15$ ) to evaluate obstructive and irritative symptoms separately.

The levels of TG, FBS, HDL, and microalbumin were measured by enzyme assays. The PSA level was measured by enzyme-linked immunosorbent assay with an immuno-enzymatic kit. The volume of the prostate was measured on the basis of the ellipsoid formula (height $x$ length $x$ width $\times \pi / 6)$ [19].

The patients were repeatedly visited on a monthly basis, evaluating clinical progression, medication compliance, and complications. The IPSS was documented again by urologists after 3 months. After enrolling 100 patients, statistical analysis was performed. The urologists were completely unaware of the MetS status of the patients.

\section{Inclusion and exclusion criteria}

Inclusion criteria were as follows: IPSS of 8 to 19 ; no secondary complications of BPH, including hydronephrosis, azotemia, urinary infection, hematuria, or bladder stone; obvious DM (FBS $\geq 126 \mathrm{mg} / \mathrm{dL}$ ); no signs or symptoms of neurogenic bladder, including low anal tonus, paresthesia, low force or disorder in lower limb reflexes, and no previous history of neurologic disorders; no chronic use of anticholinergics, diuretics, or psychotropic drugs; no history of urethritis or urethral stenosis; and no obvious proteinuria.

Exclusion criteria were as follows: dispensing with the study by the patient, stopping the medical treatment by the patient for any reason, nonresponders to medical treatment or occurrence of secondary complications of BPH during treatment, short-term weight changes, and appearance of severe hypertriglyceridemia or overt DM.

\section{Statistical analysis and ethics}

In the descriptive analysis, we used means and SDs for quantitative variables and frequency for ordinal and categorical variables. In the inferential analysis, for comparison of deterministic factors of MetS in patients, we used relative risk and $95 \%$ confidence interval. For comparison of $\mathrm{BPH}$ indices in patients with and without MetS before and after treatment, we used multivariate analysis of covariance for eliminating the confounder effect of other variables while assessing all related symptoms of BPH at once (the word multivariate is used for this aim).

In this study, the principles of the Helsinki Declaration were followed.

\section{RESULTS}

Of the 100 enrolled patients, 47 had MetS and 53 were without MetS (Fig. 1). The patients' mean \pm SD age was $62.5 \pm 9.6$ years. Mean PSA and mean prostate volume were $1.8 \pm 1.4$ $\mathrm{ng} / \mathrm{mL}$ and $51.4 \pm 2.7 \mathrm{~mL}$, respectively. Regarding the PSA level, the difference between patients with and without MetS was not statistically significant ( $p=0.348$ ), whereas the mean volume of the prostate was significantly higher in MetS patients $(57 \pm 32.65 \mathrm{~mL}$ compared with $46.00 \pm 20.19$ $\mathrm{mL}, \mathrm{p}=0.036$ ). The components of MetS in patients with and without this syndrome are compared in Table 1. As shown in the table, for all components (except for systolic and diastolic blood pressure), there were significant differences between patients with MetS and those without MetS, which indicates that the patients' categorization before treatment was acceptable.

As shown in Table 2, the IPSS and its components in patients with and without MetS showed no significant differences (except for PVR) before treatment. However, the differences became statistically significant after treatment. The decrease in IPSS components was much less in patients with MetS than in patients without MetS. Whereas the control group demonstrated an 11-unit reduction in IPSS, those with MetS had only a 6-unit reduction in the symptom score $(\mathrm{p}<0.001)$. This finding denotes that MetS negatively affected the clinical response of $\mathrm{BPH}$ to medical treatment. Prostate volume did not have a significant ef-

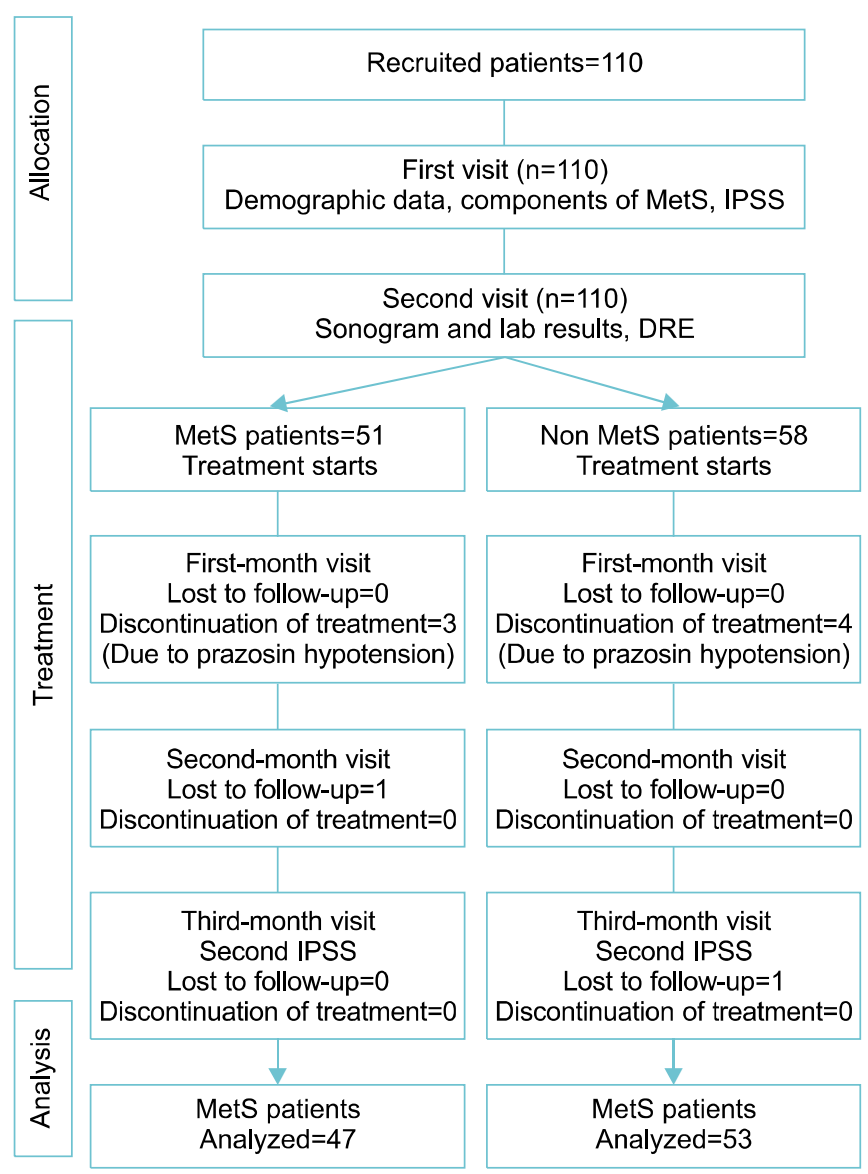

FIG. 1. Details of recruitments, visits, loss to follow-up and analyzed cases in the groups with and without metabolic syndrome. MetS, metabolic syndrome; IPSS, International Prostate Symptom Score; DRE, digital rectal examination. 
TABLE 1. Components of metabolic syndrome in patients with $\left(\mathrm{MetS}^{+}\right)$and without $\left(\mathrm{MetS}^{-}\right)$metabolic syndrome before treatment

\begin{tabular}{|c|c|c|c|c|}
\hline \multirow{2}{*}{ Component } & \multicolumn{2}{|c|}{ No. $(\%)$} & \multirow{2}{*}{ p-value } & \multirow{2}{*}{$\mathrm{RR}(95 \% \mathrm{CI})$} \\
\hline & MetS $^{+}$ & MetS $^{-}$ & & \\
\hline $\mathrm{SBP}(\mathrm{mmHg})$ & & & 0.084 & $1.80(0.90-3.58)$ \\
\hline$\geq 140$ & $16(61.5)$ & $10(38.5)$ & & \\
\hline$<140$ & $31(41.9)$ & $43(58.1)$ & & \\
\hline DBP (mmHg) & & & 0.081 & $1.88(0.90-3.88)$ \\
\hline$\geq 90$ & $15(62.5)$ & $9(37.5)$ & & \\
\hline$<90$ & $32(42.1)$ & $44(57.9)$ & & \\
\hline $\mathrm{TG}(\mathrm{mg} / \mathrm{dL})$ & & & 0.001 & $4.41(2.58-7.51)$ \\
\hline$\geq 150$ & $43(79.6)$ & $11(20.4)$ & & \\
\hline$<150$ & $4(8.7)$ & $42(91.3)$ & & \\
\hline HDL (mg/dL) & & & 0.002 & $3.38(1.46-7.80)$ \\
\hline$\leq 35$ & $18(75.0)$ & $6(25.0)$ & & \\
\hline$>35$ & $29(38.2)$ & $47(61.8)$ & & \\
\hline FBS (mg/dL) & & & $<0.001$ & $6.60(3.28-13.28)$ \\
\hline$\geq 100$ & $41(85.4)$ & $7(14.6)$ & & \\
\hline$<100$ & $6(11.5)$ & $46(88.5)$ & & \\
\hline Mircoalbuminuria $(\mathrm{mg} / \mathrm{m})$ & & & 0.038 & $2.44(1.00-5.91)$ \\
\hline$\geq 20$ & $13(68.4)$ & $6(31.6)$ & & \\
\hline$<20$ & $34(42.0)$ & $47(58.0)$ & & \\
\hline Waist circumference $(\mathrm{cm})$ & & & $<0.001$ & $2.30(1.69-3.13)$ \\
\hline$\geq 85$ & $47(67.1)$ & $23(32.9)$ & & \\
\hline$<85$ & $0(0)$ & $30(100)$ & & \\
\hline
\end{tabular}

RR, relative risk; CI, confidence interval; SBP, systolic blood pressure; DBP, diastolic blood pressure; TG, triglyceride; HDL, high-density lipoprotein; FBS, fasting blood sugar.

TABLE 2. MANCOVA results (multivariate and univariate significant differences) comparing symptoms of BPH patients with (MetS $\mathrm{S}^{+}$) and without $\left(\mathrm{MetS}^{-}\right)$metabolic syndrome before and after treatment

\begin{tabular}{|c|c|c|c|}
\hline \multirow{2}{*}{ Component } & \multicolumn{2}{|c|}{ Mean \pm SD } & \multirow{2}{*}{$\mathrm{p}$-value } \\
\hline & $\mathrm{MS}^{+}$ & $\mathrm{MS}^{-}$ & \\
\hline \multicolumn{4}{|l|}{ Before treatment } \\
\hline Overall MANCOVA & & & 0.278 \\
\hline IPSS & $16.95 \pm 8.54$ & $16.81 \pm 7.01$ & 0.615 \\
\hline Irritative score & $7.34 \pm 4.24$ & $6.90 \pm 4.04$ & 0.604 \\
\hline Obstructive score & $9.53 \pm 5.50$ & $9.13 \pm 5.29$ & 0.701 \\
\hline PVR & $2.76 \pm 1.91$ & $1.96 \pm 1.90$ & 0.038 \\
\hline Frequency & $2.34 \pm 1.78$ & $2.41 \pm 1.80$ & 0.845 \\
\hline Intermittency & $2.59 \pm 1.84$ & $2.52 \pm 1.81$ & 0.849 \\
\hline Urgency & $2.00 \pm 1.95$ & $1.64 \pm 1.89$ & 0.362 \\
\hline Force & $2.55 \pm 1.66$ & $2.88 \pm 1.58$ & 0.316 \\
\hline Hesitancy & $1.44 \pm 1.81$ & $1.71 \pm 1.87$ & 0.477 \\
\hline Nocturia & $3.10 \pm 1.86$ & $2.84 \pm 1.53$ & 0.454 \\
\hline \multicolumn{4}{|l|}{ After treatment } \\
\hline Overall MANCOVA & & & $<0.001$ \\
\hline IPSS & $11.00 \pm 7.99$ & $5.67 \pm 4.61$ & $<0.001$ \\
\hline Irritative score & $4.41 \pm 3.72$ & $2.11 \pm 2.14$ & 0.001 \\
\hline Obstructive score & $6.89 \pm 4.86$ & $2.75 \pm 2.36$ & $<0.001$ \\
\hline PVR & $2.04 \pm 1.65$ & $0.66 \pm 1.07$ & $<0.001$ \\
\hline Frequency & $1.14 \pm 1.45$ & $0.77 \pm 1.01$ & 0.138 \\
\hline Intermittency & $2.08 \pm 1.63$ & $0.96 \pm 1.17$ & $<0.001$ \\
\hline Urgency & $1.06 \pm 1.49$ & $0.28 \pm 0.60$ & 0.001 \\
\hline Force & $1.53 \pm 1.65$ & $0.90 \pm 1.04$ & 0.021 \\
\hline Hesitancy & $0.85 \pm 1.39$ & $0.30 \pm 0.60$ & 0.011 \\
\hline Nocturia & $2.34 \pm 1.64$ & $1.07 \pm 1.12$ & 0.001 \\
\hline
\end{tabular}

MANCOVA, multivariate analysis of covariance; BPH, benign prostatic hyperplasia; SD, standard deviation; IPSS, International Prostate Symptom Score; PVR, postvoiding residue. 
fect on any BPH signs or symptoms except for the IPSS total score before treatment $(\mathrm{p}=0.042)$. After control for prostate volume and age, neither BPH signs and symptoms nor IPSS differed significantly between cases with MetS and those without MetS before treatment.

Prostate volume had a significant effect on nocturia $(\mathrm{p}=0.001)$, irritative score $(\mathrm{p}=0.003)$, and IPSS $(\mathrm{p}=0.001)$ after treatment. After control for prostate volume and age, IPSS $(\mathrm{p}<0.001)$ and all BPH signs and symptoms (except frequency) were significantly different between cases with and without MetS after treatment: irritative score ( $p=0.006)$, obstructive score $(p<0.001)$, PVR $(p<0.001)$, intermittency $(p<0.001)$, urgency $(p=0.003)$, force $(p=0.023)$, hesitancy ( $\mathrm{p}=0.033)$, and nocturia $(\mathrm{p}<0.001)$.

Regarding the components of MetS separately, the levels of TG ( $p<0.001)$, FBS ( $p=0.001)$, and waist circumference $(\mathrm{p}=0.028)$ significantly affected the clinical progression of $\mathrm{BPH}$, whereas microalbumin $(\mathrm{p}=0.088)$, systolic blood pressure $(\mathrm{p}=0.143)$, diastolic blood pressure $(\mathrm{p}=0.435)$, and HDL ( $p=0.833$ ) showed no significant impact.

\section{DISCUSSION}

This study showed that MetS could negatively affect the response to medical treatment of BPH. As expected, the frequency of MetS in our sample population was high (47\%). Among the IPSS components, the most affected symptoms were PVR, intermittency, nocturia, and total obstructive symptoms. In other words, in patients with $\mathrm{BPH}$ who also had MetS, medical treatment had no effect on these symptoms. A UK study by Kupelian et al. [15] showed that the presence of MetS worsened the symptoms of BPH, including PVR, intermittency, decreased force, and hesitancy, and the overall response to treatment was poor. These results imply that MetS worsens the obstructive symptoms of BPH and also impedes the reduction in symptoms after routine treatment.

The results of our study showed that the PSA level was not significantly different between MetS patients and patients without MetS. However, a study in South Korea showed that there was a negative and significant correlation between the presence of MetS and the PSA level in healthy men without BPH. Similar results were reported by Jeong et al. [20] and Parekh et al. [21]. In a study by Park et al. [22] on healthy police officers, after adjustment for age and testosterone level, no significant correlation was shown between MetS and BPH, considering volume of the prostate and prostate involvement symptoms.

All these investigations were on healthy and young to middle-aged men. Because the PSA level has a strong correlation with the base testosterone level, any comments on the association between MetS and PSA are affected by confounding factors, and adjustments for age and testosterone level are necessary in future studies.

In our patients, prostate volume differed significantly between patients with and without MetS. After omitting the confounding effect of volume size, medication was more effective in cases without MetS. This result suggests that the effect of prazosin and finasteride is more significant in cases without MetS irrespective of prostate volume. Such an effect cannot be attributed to primary prostate volume. The results of a study in South Korea also showed that the volume of the prostate was significantly higher in MetS patients than in those without MetS. However, the PSA level did not differ significantly between these groups and the correlation between prostate volume and BMI was much stronger than for other components in MetS [23]. Another study in South Korea by Koo et al. [24] also reported similar results. All these studies imply the importance of MetS in increased prostate volume and its consequences.

Among the MetS components, TG showed the strongest correlation with disease severity and impediment in $\mathrm{BPH}$ medical treatment. Some previous studies have demonstrated that increased TG is one of the most important components of MetS and could affect the prognosis of BPH $[4,9,25]$. Furthermore, hypertriglyceridemia has also been implicated as a predisposing factor in prostate cancer [26]. It seems that educating patients about the risks associated with hypertriglyceridemia and recommendations for avoidance of saturated fats and weight reduction can enhance the chances of success in BPH treatment.

This study showed that among MetS components, increased FBS also correlated with disease severity and lower response in $\mathrm{BPH}$ treatment. Previous studies including those by Ozden et al. [4] in Turkey and Rohrmann et al. [14] in the United States have reported similar results. Some have claimed that hyperglycemia has a positive correlation with prostate volume $[6,25,27]$. It could be concluded that high FBS and DM are negative prognostic factors in the clinical progression of BPH.

This study showed that waist circumference has a statistically significant impact on $\mathrm{BPH}$ treatment. Parsons et al. [5] in the United States showed that there is a positive correlation between obesity and the occurrence of $\mathrm{BPH}$. Moreover, Laukkanen et al. [28] in Finland showed that gaining weight and obesity have a significant correlation with prostate cancer. These results confirm the important role of obesity in benign or malignant tumors of the prostate. Consequently, applying modifications such as weight reduction and maintaining the FBS level within the normal range can increase the chance of success in the medical treatment of BPH. Executing comprehensive national programs including educational panels, prevention of obesity in society, and DM treatment can be effective in this field.

In this study, although the correlation between high blood pressure and BPH was positive, it was not statistically significant $(\mathrm{p}=0.08)$. Rohrmann et al. [14] showed that there was a positive correlation between hypertension and lower urinary tract symptoms. On the other hand, Martin et al. [26] in Norway stated that among the MetS components, only high blood pressure had a significant correlation with the mortality of prostate cancer. Wallner et al. [12] corroborated the results about the relationship of high 
blood pressure with prostate cancer. Although the correlation of hypertension and $\mathrm{BPH}$ has rarely been mentioned in previous articles, multiple studies introduced high blood pressure as one of the risk factors in prostate cancer.

This study showed that HDL had no impact on response to treatment. Some studies reported a strong correlation between LDL and the occurrence of BPH $[3,25]$. These results could corroborate the theory claiming that cardiovascular disease risk factors may play a role in the pathogenesis and commencement of $\mathrm{BPH}$ and prostate cancer.

Our data revealed that microalbuminuria was not statistically related to the reduction of symptoms, but with a p-value of 0.08 , a larger sample size might have proved it to be a prognostic factor.

Considering the prognosis of response to medical treatment or the need for invasive therapies, we should not discount factors other than MetS, and more comprehensive studies are needed. For MetS, other standard criteria such as the National Cholesterol Education Program's Third Adult Treatment Panel or the European Group for the Study of Insulin Resistance [29,30] can be used. However, with the relative correlation of urinary microalbumin and response rate that we found, the WHO criteria seem to be more suitable for future studies. Regarding the mediocre sample size and short-term follow-up of this study, larger controlled trials with long-term follow-up for the rate of secondary complications and the need for surgery are recommended.

The type of analysis in the present study is completely new. None of the previous studies have considered all BPH symptoms at once by use of multivariate analysis of covariance. Similarly, none of them omitted the confounder effect of prostate volume, albeit some of them due to the nonsignificant difference in prostate volume between cases with and without MetS.

\section{CONCLUSIONS}

This study showed that MetS could negatively affect the response to medical treatment of $\mathrm{BPH}$. Therefore, it is necessary to consider MetS in selecting patients with $\mathrm{BPH}$ for drug therapy.

\section{CONFLICTS OF INTEREST}

The authors have nothing to disclose.

\section{ACKNOWLEDGMENTS}

We appreciate all the patients who participated in this study and the hospital staff who cooperated with us in many administrative issues.

\section{REFERENCES}

1. Tanagho EA, McAninch JW. Smith's general urology. New York: McGraw-Hill Medical; 2008.

2. Cruz F, Desgrandchamps F. New concepts and pathophysiology of lower urinary tract symptoms in men. Eur Urol Suppl 2010;9: 472-6.

3. Parsons JK, Bergstrom J, Barrett-Connor E. Lipids, lipoproteins and the risk of benign prostatic hyperplasia in community-dwelling men. BJU Int 2008;101:313-8.

4. Ozden C, Ozdal OL, Urgancioglu G, Koyuncu H, Gokkaya S, Memis A. The correlation between metabolic syndrome and prostatic growth in patients with benign prostatic hyperplasia. Eur Urol 2007;51:199-203.

5. Parsons JK, Sarma AV, McVary K, Wei JT. Obesity and benign prostatic hyperplasia: clinical connections, emerging etiological paradigms and future directions. J Urol 2013;189(1 Suppl): S102-6.

6. Yim SJ, Cho YS, Joo KJ. Relationship between metabolic syndrome and prostate volume in Korean men under 50 years of age. Korean J Urol 2011;52:390-5.

7. Sarma AV, Wei JT, Jacobson DJ, Dunn RL, Roberts RO, Girman CJ, et al. Comparison of lower urinary tract symptom severity and associated bother between community-dwelling black and white men: the Olmsted County Study of Urinary Symptoms and Health Status and the Flint Men's Health Study. Urology 2003;61: 1086-91.

8. De Nunzio C, Aronson W, Freedland SJ, Giovannucci E, Parsons JK. The correlation between metabolic syndrome and prostatic diseases. Eur Urol 2012;61:560-70.

9. Tewari R, Prabhat P, Natu S, Dalela D, Goel A, Goel M, et al. Association of benign prostatic hyperplasia (BPH) with the metabolic syndrome (MS) and its components: a growing dilemma. $\mathrm{J}$ Mens Health 2011;8:66-71.

10. Gogia A, Agarwal PK. Metabolic syndrome. Indian J Med Sci 2006;60:72-81.

11. Ford ES, Giles WH, Dietz WH. Prevalence of the metabolic syndrome among US adults: findings from the third National Health and Nutrition Examination Survey. JAMA 2002;287:356-9.

12. Wallner LP, Morgenstern H, McGree ME, Jacobson DJ, St Sauver JL, Jacobsen SJ, et al. The effects of metabolic conditions on prostate cancer incidence over 15 years of follow-up: results from the Olmsted County Study. BJU Int 2011;107:929-35.

13. Park HS, Oh SW, Cho SI, Choi WH, Kim YS. The metabolic syndrome and associated lifestyle factors among South Korean adults. Int J Epidemiol 2004;33:328-36.

14. Rohrmann S, Smit E, Giovannucci E, Platz EA. Association between markers of the metabolic syndrome and lower urinary tract symptoms in the Third National Health and Nutrition Examination Survey (NHANES III). Int J Obes (Lond) 2005;29: 310-6.

15. Kupelian V, McVary KT, Kaplan SA, Hall SA, Link CL, Aiyer LP, et al. Association of lower urinary tract symptoms and the metabolic syndrome: results from the Boston area community health survey. J Urol 2013;189(1 Suppl):S107-14.

16. Han JH, Chang IH, Ahn SH, Kwon OJ, Bang SH, Choi NY, et al. Association between serum prostate-specific antigen level, liver function tests and lipid profile in healthy men. BJU Int 2008; 102:1097-101.

17. Mehrdad M, Hosainpanah F, Azizi F. Prevalence of metabolic syndrome among 3-9 years old children inTehran Lipid and Glucose Study. Pejouhesh 2006;30:337-46.

18. Sadrbafoghi SM, Salari M, Rafiee M, Namayandeh SM, Abdoli AM, Karimi M, et al. Prevalence and criteria of metabolic syndrome in an urban population:Yazd Healthy Heart Project. Tehran Univ Med J 2006;64:90-6.

19. Park SB, Kim JK, Choi SH, Noh HN, Ji EK, Cho KS. Prostate volume measurement by TRUS using heights obtained by transaxial 
and midsagittal scanning: comparison with specimen volume following radical prostatectomy. Korean J Radiol 2000;1:110-3.

20. Jeong IG, Hwang SS, Kim HK, Ahn H, Kim CS. The association of metabolic syndrome and its components with serum prostate-specific antigen levels in a Korean-screened population. Cancer Epidemiol Biomarkers Prev 2010;19:371-80.

21. Parekh N, Lin Y, Marcella S, Kant AK, Lu-Yao G. Associations of lifestyle and physiologic factors with prostate-specific antigen concentrations: evidence from the National Health and Nutrition Examination Survey (2001-2004). Cancer Epidemiol Biomarkers Prev 2008;17:2467-72.

22. Park YW, Min SK, Lee JH. Relationship between lower urinary tract symptoms/benign prostatic hyperplasia and metabolic syndrome in Korean men. World J Mens Health 2012;30:183-8.

23. Jang TH, Son JH, Kim JI, Jang SH. Metabolic Syndrome and benign prostatic hyperplasia: a study focused on the correlation between metabolic syndrome factors and prostate volume and prostate-specific antigen. Korean J Urol 2008;49:986-91.

24. Koo KC, Cho KS, Kang EM, Kwon SW, Hong SJ. The relationship between metabolic syndrome and prostate volume in men over sixties who underwent prostate health check-up. Korean J Urol 2008;49:813-7.

25. Nandeesha H, Koner BC, Dorairajan LN, Sen SK. Hyperinsuli- nemia and dyslipidemia in non-diabetic benign prostatic hyperplasia. Clin Chim Acta 2006;370:89-93.

26. Martin RM, Vatten L, Gunnell D, Romundstad P, Nilsen TI. Components of the metabolic syndrome and risk of prostate cancer: the HUNT 2 cohort, Norway. Cancer Causes Control 2009; 20:1181-92

27. Kim WT, Yun SJ, Choi YD, Kim GY, Moon SK, Choi YH, et al. Prostate size correlates with fasting blood glucose in non-diabetic benign prostatic hyperplasia patients with normal testosterone levels. J Korean Med Sci 2011;26:1214-8.

28. Laukkanen JA, Laaksonen DE, Niskanen L, Pukkala E, Hakkarainen A, Salonen JT. Metabolic syndrome and the risk of prostate cancer in Finnish men: a population-based study. Cancer Epidemiol Biomarkers Prev 2004;13:1646-50.

29. Baldeweg SE, Golay A, Natali A, Balkau B, Del Prato S, Coppack SW. Insulin resistance, lipid and fatty acid concentrations in 867 healthy Europeans. European Group for the Study of Insulin Resistance (EGIR). Eur J Clin Invest 2000;30:45-52.

30. Tan CE, Ma S, Wai D, Chew SK, Tai ES. Can we apply the National Cholesterol Education Program Adult Treatment Panel definition of the metabolic syndrome to Asians? Diabetes Care 2004;27:1182-6. 